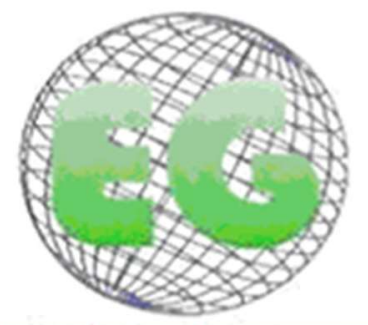

ISSN 1695-6141 $\mathbf{N}^{\circ} 62$

\title{
ORIGINALES
}

\section{Calidad de vida relacionada con la salud en personas con hipertensión y diabetes mellitus}

Quality of life related to health in people with hypertension and diabetes mellitus

\author{
Dora Inés Parra ${ }^{1}$ \\ Luis Alberto López Romero² \\ Lina María Vera Cala ${ }^{3}$
}

1 Enfermera, Magister Enfermería. Estudiante de Doctorado Programa de Enfermería Clínica y Comunitaria, Universidad de Valencia, España. Profesora, Escuela de Enfermería, Universidad Industrial de Santander, Bucaramanga, Colombia. doiparra@uis.edu.co

2 Enfermero, Magister en Epidemiología. Fundación Cardiovascular de Colombia.

3 Médica, Magíster, PhD. Profesora del Departamento de Salud Pública, Escuela de Medicina, Universidad Industrial de Santander, Bucaramanga, Colombia.

\section{https://doi.org/10.6018/eglobal.423431}

Recibido: $18 / 04 / 2020$

Aceptado: 4/09/2020

\section{RESUMEN:}

Objetivo: Determinar los factores sociodemográficos y clínicos asociados a la Calidad de Vida relacionada con la salud (CVRS) en personas con hipertensión arterial y diabetes mellitus tipo 2.

Métodos: Estudio analítico de corte transversal en una muestra de 184 usuarios de un programa de control de riesgo cardiovascular en Bucaramanga (Colombia). Se utilizó el instrumento EQ-5D-3L para evaluar la calidad de vida. Un modelo de regresión lineal múltiple fue llevado a cabo usando como desenlace la Escala Visual Análoga y como posibles predictores las dimensiones del EQ-5D, ajustando por edad, sexo, grupo de intervención (variable instrumental) y variables clínicas como la patología de base y el control de la misma.

Resultados: La mediana de edad fue 63 años, 73,3\% mujeres, 88,0\% nivel socioeconómico bajo; mediana de Presión Arterial Sistólica de 130 mmHg $(116,0-145,0)$ y de HbA1c 5,7\% (5,4-6,2) en la población general, hallándose diferencias estadísticamente significativas entre los grupos de patología. La mediana de la Escala Visual Análoga fue de 80(Q1:59-Q3:95) puntos, sin diferencias estadísticamente significativas entre los grupos de patología. Manifestar dolor/malestar, tener problemas en las actividades cotidianas y el no control de la hipertensión arterial disminuyó la percepción de la calidad de vida relacionada con la salud, en 7,5, 18,1 y 7,3 puntos, respectivamente.

Conclusiones: La CVRS, en general, fue alta. Esta fue mayor en las personas con diabetes mellitus tipo 2. Factores relacionados con la funcionalidad y control de la enfermedad fueron asociados con una menor percepción de la CVRS.

Palabras clave: Calidad de Vida Relacionada con Salud, Hipertensión, Diabetes mellitus.

\section{ABSTRACT:}

Objective: To determine the sociodemographic and clinical factors associated with health-related Quality of Life (HRQoL) among people with hypertension and type 2 diabetes mellitus. 
Methods: Analytical cross-sectional study in a sample of 184 users of a cardiovascular risk control program in Bucaramanga (Colombia). The EQ-5D-3L instrument was used to assess quality of life. A multiple linear regression model was carried out using the Visual Analog Scale as the outcome and the dimensions of the EQ-5D as possible predictors, adjusting for age, sex, intervention group (instrumental variable), and clinical variables such as the disease and its control.

Results: The median of age was 63 years, $73.3 \%$ women, $88.0 \%$ low socioeconomic level; Median Systolic Blood Pressure of $130 \mathrm{mmHg}(116.0-145.0)$ and HbA1c 5.7\% (5.4-6.2) in the general population, showing statistically significant differences between the pathology groups. The median of Visual Analog Scale was 80(Q1: 59-Q3: 95) points, with no statistically significant differences between the pathology groups. Manifesting pain/discomfort, problems with usual activities and lack of hypertension control decreased the perception of HRQoL, by 7.5, 18.1 and 7.3 points, respectively.

Conclusions: The HRQoL in general was high. It was higher among people with type 2 diabetes mellitus. Factors related to the functionality and control of the disease were associated with a lower perception HRQoL.

Key words: Health Related Quality of Life, HRQoL, Hypertension, Diabetes mellitus.

\section{INTRODUCCIÓN}

La calidad de vida es un concepto ampliamente estudiado, complejo y multifactorial que abarca aspectos internos y externos de cada individuo ${ }^{(1,2)}$. En años recientes ha surgido gran interés en evaluar la Calidad de Vida Relacionada con Salud (CVRS) tanto de individuos sanos como en aquellos afectados por determinadas patologías ${ }^{(3-}$ 5), debido a la gran utilidad de este indicador en la asignación de recursos y evaluación de políticas de salud.

Por otro lado, enfermedades crónicas como HTA y DM2 están surgiendo debido al envejecimiento de la población, siendo cada vez frecuente encontrar personas con más de una de estas patologías ${ }^{(6,7)}$, lo cual afecta altamente su calidad de vida.

Tradicionalmente, se han evaluado estas condiciones en términos de

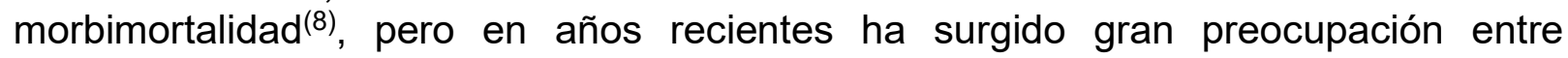
investigadores y tomadores de decisiones en la estimación de la CVRS de personas que padecen estas patologías, dada su afectación no solo por la presencia de la enfermedad, sino también por el tratamiento prescrito y los cambios en los estilos de vida que deben asumir ${ }^{(9)}$.

La HTA puede disminuir la calidad de vida del paciente debido a las múltiples complicaciones en órganos blancos y a los efectos secundarios de los medicamentos antihipertensivos, por ello, es importante controlar HTA para reducir significativamente el riesgo de complicaciones y por ende mejorar la calidad de vida de estos pacientes $^{(9)}$. De otro lado, DM2 afecta la calidad de vida de los pacientes ${ }^{(6)}$, por su gran carga financiera y social ${ }^{(10,11)}$, que no solo impacta su salud y bienestar, sino también sus hábitos y estilos de vida ${ }^{(12)}$.

Existen algunos factores relacionados con el aumento o la disminución en la percepción de la CVRS en pacientes con HTA y DM2, tales como sexo, edad, bajo control de la enfermedad, tipo de enfermedad, bajo nivel educativo, dependencia de los cuidadores, no-adherencia al tratamiento, y alteraciones de movilidad, entre otros (13-15). 
El contexto anterior hace necesario evaluar la CVRS de los afectados por HTA y DM2 y los factores que pueden afectarla, para intervenir aspectos potencialmente modificables para mejorar su estado de salud. Al respecto, existen instrumentos que permiten medir de forma genérico la CVRS, como el EQ-5D-3L, que determina la auto-percepción del paciente de su estado de salud a través de cinco dimensiones: movilidad, autocuidado, actividades cotidianas, dolor/malestar, ansiedad/depresión $(16,17)$.

EQ-5D-3L es una herramienta "aplicable a un amplio rango de afecciones de salud y tratamientos, que proporciona un perfil descriptivo simple y un valor de índice único para el estado de salud, utilizable para evaluación clínica y financiera de la atención médica, y también para encuestas de salud poblacional" $(16,17)$.

El objetivo de este estudio fue determinar los factores sociodemográficos y clínicos asociados con la CVRS, en pacientes con HTA y DM2 de un programa de atención primaria que participaron en un ensayo clínico aleatorizado (ECA).

\section{MATERIALES Y MÉTODOS}

\section{Diseño y población}

Estudio analítico, de corte transversal en una muestra de 184 personas que participaron en un ensayo clínico aleatorizado (ECA) con el objetivo de establecer la eficacia de una intervención educativa para aumentar la adherencia al tratamiento. Los participantes tenían diagnóstico médico de hipertensión o DM2 y formaban parte de un programa de control de riesgo cardiovascular en un centro de atención primaria de Bucaramanga (Colombia) entre mayo de 2016 y septiembre de $2017{ }^{(18)}$.

\section{Instrumento y mediciones}

Todos los datos fueron obtenidos a lo largo del ECA. Este estudio analizó las siguientes variables:

Calidad de vida relacionada con salud CVRS (variable dependiente), definida como la medición descriptiva, evaluativa, y predictiva del estado de salud de un individuo; fue evaluada utilizando el cuestionario EQ-5D-3L, una herramienta para evaluar la condición de salud de las personas.

El cuestionario EQ-5D-3L es un instrumento genérico de dos partes. La primera parte se basa en una clasificación multi-atributos que cubre cinco dimensiones: movilidad, autocuidado, actividades cotidianas, dolor/malestar y ansiedad/depresión, y cada una de ellas tiene tres niveles de severidad: sin problemas, algunos o moderados problemas, y problemas severos, calificados respectivamente por 1, 2, o 3 puntos, donde a mayor puntuación, mayores los problemas en las diferentes dimensiones del cuestionario, y peor la calidad de vida relacionada con salud (19). Un solo dígito expresa el nivel seleccionado para esa dimensión específica.

Por ejemplo, "11111" indica "sin problemas" en cualquiera de las cinco dimensiones, mientras que "21121" indica algunos o moderados problemas en la dimensión movilidad, sin problemas en la dimensión autocuidado y actividades cotidianas, 
algunos o moderados problemas en la dimensión dolor/malestar, y sin problemas en la dimensión ansiedad/depresión. Asimismo, un resultado de 21133 indica algunos o moderados problemas en la dimensión movilidad, sin problemas en la dimensión autocuidado y actividades cotidianas, y severos problemas en las dimensiones dolor/malestar y ansiedad/depresión.

La combinación de los niveles de gravedad con todas las dimensiones define el estado de salud, los cuales corresponden a 243 posibilidades que pueden utilizarse como perfiles ${ }^{(20,21)}$. Los estados de salud para cada una de las 5 dimensiones se convierten en un índice de estado de salud ponderado, asignando los pesos de preferencia del EQ-5D obtenidos de la muestra de la población general. Estos pesos fluctúan entre 1, (Salud total) y 0 (Muerte) ${ }^{(22)}$.

La segunda parte es una Escala Análoga Visual (VAS) que varía entre cero (0), como peor estado de salud, y 100, como estado óptimo de salud, obteniéndose un índice de CVRS. Los participantes debían marcar el punto en la línea vertical que mejor reflejaba la valoración de su actual estado global de salud. Refleja el estado general de salud del paciente, representando una medida más integral que el índice EQ$5 D^{(14)}$.

Variables clínicas del control de la enfermedad (Variables independientes): La presión arterial sistólica (PAS) se obtuvo a través de medición casual y la hemoglobina glicosilada (HbA1c) por medio de muestra de sangre ${ }^{(18)}$. Control de HTA se definió como tener PAS $<140 \mathrm{mmHg}{ }^{(23)}$. Igualmente, control de diabetes se definió como tener $\mathrm{HbA} 1 \mathrm{c}<7,0 \%$ en pacientes independientes y libres de otras comorbilidades mayores $(24,25)$.

Otras variables incluidas en el estudio fueron la enfermedad del paciente (HTA, DM2 o ambas) y el grupo del ECA (Intervenido o de control como variable instrumental de ajuste en el modelo final).

Variables sociodemográficas (Variables independientes): tales como edad en años, sexo y nivel socioeconómico.

\section{Procedimiento de recolección de datos}

Para obtener el estado de salud actual de los participantes, se tomó los registros del cuestionario EQ-5D-3L que fue aplicado durante la última medición del ECA al final del mes 12. Las demás, variables de interés (variables sociodemográficas, clínicas y del grupo de intervención) también fueron tomadas del estudio original.

\section{Análisis estadístico}

Se efectuó análisis descriptivo de las variables sociodemográficas, clínicas y de la CVRS, estratificando según la patología del paciente. Las variables continuas se describieron por medio de medianas y su correspondiente primer y tercero cuartiles dado que no mostraban una distribución normal según las pruebas estadísticas Shapiro Wilk y la prueba Sktest. Las variables nominales politómicas fueron descritas como frecuencias absolutas y relativas.

El análisis descriptivo relacionado con la CVRS, consistió, primero en hallar el estado 
de salud de los participantes por dimensiones y estado de gravedad según el EQ-5D. Luego se combinaron los valores de todas las dimensiones, generándose los estados de salud posible o perfiles de la población en estudio. Posteriormente, se determinó el estado de salud global mediante el calculó de las medidas de tendencia central del VAS acompañado de sus respectivas medidas de dispersión.

Finalmente, se obtuvo el índice de valores de preferencias en cada estado de salud generados por el EQ-5D (índice EQ-5D), tomando como referencia los coeficientes para el cálculo de valores sociales descritos en España ${ }^{(3)}$. Además, se determinó los Años de Vida Ajustados por Calidad (AVAC), al multiplicar el índice EQ-5D por la edad actual del paciente al momento de la entrevista.

También se efectuó un análisis bivariado comparando la enfermedad del paciente con las medianas del VAS, el índice EQ-5D, los AVAC y las dimensiones del EQ-5D, que fueron analizadas en dos categorías: "sin problemas", y "con problemas". Para ello se utilizó la prueba Kruskal-Wallis para variables continuas y la prueba de Ji cuadrado para las variables categóricas, respectivamente.

Finalmente, se realizó un modelo de regresión lineal múltiple robusta utilizando VAS como desenlace y como posibles predictores principales las dimensiones del EQ-5D ajustando por edad, sexo, nivel socioeconómico, grupo de intervención (variable instrumental), variables clínicas como el control de la enfermedad (control de la tensión arterial y control en la hemoglobina glicosilada) y el tipo de patología del paciente (solo HTA, solo DM2 y ambos). Se consideró significativo un valor de $p$ inferior a 0,05. Todas las pruebas estadísticas se realizaron a dos colas. Los datos se analizaron utilizando el programa de estadísticas STATA, versión 14,0.

\section{Consideraciones éticas}

Este estudio fue aprobado por dos comités de ética en investigación. Los participantes dieron consentimiento por escrito para ser incluidos en el estudio original. La confidencialidad de la información se garantizó utilizando códigos numéricos en las bases de datos. Se obtuvo permiso para utilizar el instrumento EQ-5D-3L. Finalmente, el estudio cumplió la regulación nacional e internacional para ejecutar investigación en seres humanos ${ }^{(26)}$.

\section{RESULTADOS}

\section{Características de los participantes}

La Tabla 1 muestra las características sociodemográficas y clínicas. La muestra para este estudio se componía de 184 participantes, siendo la mayoría hipertensos $(n=144)$. Entre los aspectos más relevantes se destacan: una mediana de edad 63 años; la mayoría mujeres $(73,3 \%)$ y un nivel socioeconómico bajo predominante $(88,0 \%)$. Adicionalmente, se observa que no hubo diferencias estadísticamente significativas entre los participantes según patología de base para las características sociodemográficas, excepto para variables clínicas de control de la enfermedad, tales como la PAS y la HbA1c. 
Tabla 1: Características Sociodemográficas de los participantes en el estudio

\begin{tabular}{|c|c|c|c|c|c|}
\hline Variables & $\begin{array}{c}\text { Todos } \\
(n=184)\end{array}$ & $\begin{array}{c}\text { DM2 } \\
(n=23)\end{array}$ & $\begin{array}{c}\text { HTA } \\
(n=117)\end{array}$ & $\begin{array}{c}\text { DM2-HTA } \\
(n=44)\end{array}$ & $\begin{array}{c}\text { Valor } \\
\text { pł }\end{array}$ \\
\hline $\begin{array}{l}\text { Edad } \\
\text { (años) }\end{array}$ & $\begin{array}{c}63,0(56,0- \\
71,0)\end{array}$ & $\begin{array}{c}62,0(52,0- \\
69,0)\end{array}$ & $\begin{array}{c}65,0(56,0- \\
73,0)\end{array}$ & $\begin{array}{c}62,0(56,0- \\
69,0)\end{array}$ & 0,285 \\
\hline \multicolumn{6}{|l|}{ Sexo } \\
\hline Hombre & $49(26,6)$ & $5(21,7)$ & $31(26,5)$ & $13(29,5)$ & \multirow[t]{2}{*}{0,789} \\
\hline Mujer & $135(73,3)$ & $18(78,2)$ & $86(73,5)$ & $31(70,4)$ & \\
\hline \multicolumn{6}{|c|}{ Nivel socioeconómico } \\
\hline Bajo & $162(88,0)$ & $22(95,6)$ & $101(86,3)$ & $39(88,6)$ & \multirow[t]{2}{*}{0,448} \\
\hline Medio & $22(11,9)$ & $1(4,3)$ & $16(13,6)$ & $5(11,3)$ & \\
\hline \multicolumn{6}{|c|}{ Variables clínicas } \\
\hline PAS, $\mathrm{mmHg}$ & $\begin{array}{c}130(116,0- \\
145,0)\end{array}$ & $\begin{array}{c}118(107,0- \\
133,0)\end{array}$ & $\begin{array}{c}131(115,0- \\
145,0)\end{array}$ & $\begin{array}{c}132(121,0- \\
150,0)\end{array}$ & 0,008 \\
\hline $\mathrm{HbA1} c, \%$ & $5,7(5,4-6,2)$ & $7,0(6,1-9,4)$ & $5,6(5,3-5,8)$ & $6,5(5,8-7,6)$ & 0,001 \\
\hline
\end{tabular}

† Prueba de Ji cuadrado para variables categóricas y prueba Kwallis para variables continuas. Esta tabla contiene $\mathrm{n}(\%)$ para valores categóricos, mediana (primer y tercer cuartil, Q1-Q3 para variables continuas). Abreviaciones: $\mathrm{PAS}=$ Presión Arterial Sistólica; $\mathrm{HbA} 1_{\mathrm{c}}=$ Hemoglobina glicosilada.

\section{Estado de salud de los participantes según dimensiones del EQ-5D}

Entre las dimensiones de la CVRS, el "cuidado personal" fue la menos problemática a nivel general $(96,2 \%)$, y la más afectada fue "dolor/malestar" $(47,8 \%)$, sin evidencia de asociación estadísticamente significativa con la patología que padece el paciente. Hubo asociación estadísticamente significativa entre la dimensión "actividades cotidianas" y la patología base. Al observar el comportamiento de las dimensiones por patología se determinó que los pacientes con DM2 tenían el porcentaje más alto de no problemas para tres de las dimensiones, excepto para dolor/malestar y ansiedad/depresión (Tabla 2).

Tabla 2: Dimensiones del estado de salud de los participantes

\begin{tabular}{|c|c|c|c|c|c|}
\hline Dimensión & Todos & DM2 & HTA & HTA/DM2 & $\begin{array}{l}\text { Valor } \\
\text { p }\end{array}$ \\
\hline \multicolumn{6}{|l|}{ Movilidad } \\
\hline $\begin{array}{l}\text { No tengo } \\
\text { problemas para } \\
\text { caminar }\end{array}$ & $123(66,8)$ & $19(82,6)$ & $79(67,5)$ & $25(56,8)$ & \multirow[t]{2}{*}{0,100} \\
\hline $\begin{array}{l}\text { Tengo algunos } \\
\text { problemas para } \\
\text { caminar }\end{array}$ & $61(33,1)$ & $4(17,3)$ & $38(32,4)$ & $19(43,1)$ & \\
\hline \multicolumn{6}{|l|}{ Cuidado personal } \\
\hline $\begin{array}{l}\text { No tengo } \\
\text { problemas con el } \\
\text { cuidado personal }\end{array}$ & $177(96,2)$ & $23(100,0)$ & $112(95,7)$ & $42(95,4)$ & \multirow[t]{2}{*}{0,593} \\
\hline $\begin{array}{l}\text { Tengo } \\
\text { problemas para } \\
\text { lavarme o }\end{array}$ & $7(3,8)$ & $0(0,0)$ & $5(4,2)$ & $2(4,5)$ & \\
\hline
\end{tabular}




\begin{tabular}{|c|c|c|c|c|c|}
\hline vestirme & & & & & \\
\hline \multicolumn{6}{|c|}{ Actividades cotidianas } \\
\hline $\begin{array}{l}\text { No tengo } \\
\text { problemas para } \\
\text { realizar mis } \\
\text { actividades } \\
\text { cotidianas }\end{array}$ & $171(92,9)$ & $23(100,0)$ & $111(94,8)$ & $37(84,0)$ & \multirow[t]{2}{*}{0,022} \\
\hline $\begin{array}{l}\text { Tengo problemas } \\
\text { para realizar mis } \\
\text { actividades } \\
\text { cotidianas }\end{array}$ & $13(7,0)$ & $0(0,0)$ & $6(5,1)$ & $7(15,9)$ & \\
\hline \multicolumn{6}{|l|}{ Dolor/malestar } \\
\hline $\begin{array}{l}\text { No tengo dolor ni } \\
\text { malestar }\end{array}$ & $96(52,1)$ & $13(56,5)$ & $62(52,9)$ & $21(47,7)$ & \multirow[t]{2}{*}{0,758} \\
\hline $\begin{array}{l}\text { Tengo dolor y } \\
\text { malestar }\end{array}$ & $88(47,8)$ & $10(43,4)$ & $55(47,0)$ & $23(52,2)$ & \\
\hline \multicolumn{6}{|c|}{ Ansiedad/depresión } \\
\hline $\begin{array}{l}\text { No estoy ansioso } \\
\text { ni deprimido }\end{array}$ & $133(72,2)$ & $14(60,8)$ & $87(74,3)$ & $32(72,7)$ & \multirow[t]{2}{*}{0,417} \\
\hline $\begin{array}{l}\text { Estoy ansioso o } \\
\text { deprimido }\end{array}$ & $51(27,7)$ & $9(39,1)$ & $30(25,6)$ & $12(27,2)$ & \\
\hline
\end{tabular}

$¥$ Prueba Ji cuadrado para variables categóricas.

\section{Perfiles de salud de los participantes}

Con respecto a los perfiles de los estados de salud, se observa que cinco estados son los más frecuentes en la población de estudio, lo que corresponde al $69,5 \%(n=128)$, de los cuales $32,6 \%(n=60)$ pertenece al estado de "salud completa" ("11111"). En general, los participantes presentaron problemas moderados, y asignaron un puntaje de 2 a 1 o más de las cinco dimensiones. La puntuación 3, correspondiente a problemas graves, fue la más frecuente para la dimensión 5 (ansiedad/depresión) del EQ-5D-3L. No se hallaron diferencias estadísticamente significativas entre los grupos por patologías de base.

Al disgregar por patología de base se determinó que los DM2 tenían la proporción más alta del estado de "salud completa", y menor proporción de problemas moderados o graves en alguna de las dimensiones de EQ-5D-3L, excepto para los estados 21111, 21122, 11122 y 11123, donde la frecuencia fue mayor, correspondiente a problemas moderados en las dimensiones de movilidad, dolor/malestar, ansiedad/depresión, y problemas severos en la dimensión ansiedad/depresión. Las personas con HTA se distribuyeron en mayor número de estados (Tabla 3). 
Tabla 3: Perfiles de estados de salud de los participantes

\begin{tabular}{|c|c|c|c|c|c|}
\hline \multirow[t]{2}{*}{ Estados } & $\begin{array}{c}\text { Todos } \\
(n=184)\end{array}$ & $\begin{array}{c}\text { DM2 } \\
(n=23)\end{array}$ & $\begin{array}{c}\text { HTA } \\
(n=117)\end{array}$ & $\begin{array}{c}\text { HTA/DM } \\
(n=44)\end{array}$ & Valor $p$ \\
\hline & $\mathrm{n}(\%)$ & $\mathrm{n}(\%)$ & $\mathrm{n}(\%)$ & $\mathrm{n}(\%)$ & \multirow[t]{30}{*}{0,520} \\
\hline 11111 & $60(32,61)$ & $10(43,48)$ & $36(30,77)$ & $14(31,82)$ & \\
\hline 11121 & $26(14,13)$ & $3(13,04)$ & $17(14,53)$ & $6(13,64)$ & \\
\hline 21121 & $18(9,78)$ & $0(0,00)$ & $14(11,97)$ & $4(9,09)$ & \\
\hline 11112 & $14(7,61)$ & $2(8,70)$ & $11(9,40)$ & $1(2,27)$ & \\
\hline 21111 & $10(5,43)$ & $1(4,35)$ & $7(5,98)$ & $2(4,55)$ & \\
\hline 21122 & $9(4,89)$ & $2(8,70)$ & $4(3,42)$ & $3(6,82)$ & \\
\hline 11113 & $6(3,26)$ & $0(0,00)$ & $5(4,27)$ & $1(2,27)$ & \\
\hline 11122 & $5(2,72)$ & $3(13,04)$ & $1(0,85)$ & $1(2,27)$ & \\
\hline 21131 & $5(2,72)$ & $0(0,00)$ & $3(2,56)$ & $2(4,55)$ & \\
\hline 11131 & $4(2,17)$ & $0(0,00)$ & $4(3,42)$ & $0(0,00)$ & \\
\hline 11123 & $3(1,63)$ & $1(4,35)$ & $1(0,85)$ & $1(2,27)$ & \\
\hline 11221 & $2(1,09)$ & $0(0,00)$ & $2(1,71)$ & $0(0,00)$ & \\
\hline 11222 & $2(1,09)$ & $0(0,00)$ & $1(0,85)$ & $1(2,27)$ & \\
\hline 21112 & $2(1,09)$ & $0(0,00)$ & $1(0,85)$ & $1(2,27)$ & \\
\hline 21123 & $2(1,09)$ & $1(4,35)$ & $1(0,85)$ & $0(0,00)$ & \\
\hline 21211 & $2(1,09)$ & $0(0,00)$ & $0(0,00)$ & $2(4,55)$ & \\
\hline 22121 & $2(1,09)$ & $0(0,00)$ & $1(0,85)$ & $1(2,27)$ & \\
\hline 12122 & $1(0,54)$ & $0(0,00)$ & $1(0,85)$ & $0(0,00)$ & \\
\hline 21132 & $1(0,54)$ & $0(0,00)$ & $1(0,85)$ & $0(0,00)$ & \\
\hline 21133 & $1(0,54)$ & $0(0,00)$ & $1(0,85)$ & $0(0,00)$ & \\
\hline 21212 & $1(0,54)$ & $0(0,00)$ & $1(0,85)$ & $0(0,00)$ & \\
\hline 21221 & $1(0,54)$ & $0(0,00)$ & $1(0,85)$ & $0(0,00)$ & \\
\hline 21222 & $1(0,54)$ & $0(0,00)$ & $0(0,00)$ & $1(2,27)$ & \\
\hline 21223 & $1(0,54)$ & $0(0,00)$ & $0(0,00)$ & $1(2,27)$ & \\
\hline 21322 & $1(0,54)$ & $0(0,00)$ & $0(0,00)$ & $1(2,27)$ & \\
\hline 22131 & $1(0,54)$ & $0(0,00)$ & $1(0,85)$ & $0(0,00)$ & \\
\hline 22231 & $1(0,54)$ & $0(0,00)$ & $0(0,00)$ & $1(2,27)$ & \\
\hline 32133 & $1(0,54)$ & $0(0,00)$ & $1(0,85)$ & $0(0,00)$ & \\
\hline 33211 & $1(0,54)$ & $0(0,00)$ & $1(0,85)$ & $0(0,00)$ & \\
\hline
\end{tabular}

ł Prueba de Ji cuadrado; PAA= Presión Arterial Alta. Fuente: autores

\section{Estado de salud global de los participantes del estudio e índices del EQ-5D}

La valoración del estado de salud global de todos los participantes del estudio mediante el uso del VAS obtuvo una mediana de 80(Q1:59-Q3:95) puntos y al disgregar por patología de base, se observó que las personas con DM2 obtuvieron un mayor puntaje, sin diferencias estadísticamente significativas entre grupos por patología (Tabla 4).

En cuanto a los índices de valores de preferencias, se observó que el Índex EQ-5D, fue en general alto para la población en estudio, siendo mayor en las personas con DM2 y menor en la población con HTA/DM2 (Tabla 4). Con respecto a los AVAC, se 
encontró un mayor valor entre los participantes con DM2, pero, de nuevo, sin diferencias estadísticamente significativas entre los grupos de enfermedad.

Tabla 4: Indicadores del EQ-5D

\begin{tabular}{|l|c|c|c|c|c|}
\hline $\begin{array}{l}\text { Indicadores } \\
\text { EQ-5D }\end{array}$ & $\begin{array}{c}\text { Todos } \\
{ }^{*} \text { Med } \\
(\mathrm{Q} 1-\mathrm{Q} 3)\end{array}$ & $\begin{array}{c}\text { DM } \\
\text { Med } \\
(\mathrm{Q} 1-\mathrm{Q} 3)\end{array}$ & $\begin{array}{c}\text { HTA } \\
\text { Med } \\
(\mathrm{Q} 1-\mathrm{Q} 3)\end{array}$ & $\begin{array}{c}\text { HTA/DM } \\
\text { Med } \\
(\mathrm{Q} 1-\mathrm{Q} 3)\end{array}$ & $\begin{array}{c}\text { Valor } \\
\mathrm{p} \neq\end{array}$ \\
\hline VAS & $80(59-95)$ & $90(70-100)$ & $80(50-90)$ & $80(55-95)$ & 0,207 \\
\hline Índex EQ-5D- & $0,80(0,7-$ & $0,80(0,7-$ & $0,79(0,7-$ & $0,76(0,7-1,0)$ & 0,294 \\
& $1,0)$ & $1,0)$ & $1,0)$ & & \\
\hline AVAC & $49,8(40,5-$ & $52,0(43,1-$ & $50,0(40,3-$ & $47,4(39,7-$ & 0,481 \\
& $58,0)$ & $62,0)$ & $58,0)$ & $56,4)$ & \\
\hline
\end{tabular}

$¥$ Kruskal-Wallis para variables continuas. Fuente: autores.

${ }^{*}$ Med: Mediana.

\section{Factores asociados con la CVRS en personas con HTA y DM2}

En el Modelo 2, ajustado por edad, sexo y grupo de intervención, se halló que los participantes con problemas de dolor/malestar tenían en promedio 7,5 menos puntos en la VAS, comparados con los no afectados por este problema.

En cuanto al modelo 3, ajustado por edad, sexo, grupo intervenido, patología base, HTA controlada y DM2 controlado, se encontró que, las personas con problemas en las actividades cotidianas tenían en promedio 18,3 puntos menos en la VAS que los que no manifestaron tales problemas. Del mismo modo, pacientes sin control de HTA obtuvieron 7,1 puntos menos en la VAS que los que la controlaban (Tabla 5).

Tabla 5: Factores asociados con CVRS en personas con HTA y DM2

\begin{tabular}{|c|c|c|c|c|c|c|c|c|c|}
\hline \multirow{2}{*}{$\begin{array}{l}\text { Escala } \\
\text { Análoga } \\
\text { Visual } \\
\text { (VAS) }\end{array}$} & \multicolumn{3}{|c|}{ Modelo $1\left(R^{2}=22,17\right) \ddagger$} & \multicolumn{3}{|c|}{ Modelo $2\left(R^{2}=22,24\right) ¥$} & \multicolumn{3}{|c|}{$\begin{array}{c}\text { Modelo 3 } \\
\left(R^{2}=21,72\right) ¥ ¥\end{array}$} \\
\hline & $\beta$ & $\begin{array}{c}\text { Error } \\
\text { estándar }\end{array}$ & IC $95 \%$ & $\beta$ & $\begin{array}{c}\text { Error } \\
\text { están } \\
\text { dar }\end{array}$ & $\begin{array}{c}\text { IC } \\
95 \%\end{array}$ & $\beta$ & $\begin{array}{c}\text { Error } \\
\text { están } \\
\text { dar }\end{array}$ & $\begin{array}{c}\text { IC } \\
95 \%\end{array}$ \\
\hline \multicolumn{10}{|c|}{ Referencia: No tener problemas de movilidad } \\
\hline $\begin{array}{l}\text { Problemas } \\
\text { de } \\
\text { movilidad, } \\
\text { (Si) }\end{array}$ & $-2,1$ & 3,7 & $-9,5 ; 5,2$ & $-2,9$ & 3,7 & $\begin{array}{c}-10,3 \\
4,4\end{array}$ & $-3,0$ & 3,7 & $\begin{array}{c}-10,4 \\
4,4\end{array}$ \\
\hline \multicolumn{10}{|c|}{ Referencia: Sin problemas con el cuidado personal } \\
\hline $\begin{array}{l}\text { Problemas } \\
\text { con } \\
\text { cuidado } \\
\text { personal, } \\
\text { (si) }\end{array}$ & $-13,1$ & 13,7 & $\begin{array}{c}-40,2 \\
13,9\end{array}$ & $-12,4$ & 13,2 & $\begin{array}{l}-38,6- \\
13,7\end{array}$ & $\overline{11,7}$ & 13,9 & $\begin{array}{l}-39,1 ; \\
15,7\end{array}$ \\
\hline
\end{tabular}




\begin{tabular}{|c|c|c|c|c|c|c|c|c|c|}
\hline \multicolumn{10}{|c|}{ Referencia: Sin problemas con las actividades cotidianas } \\
\hline $\begin{array}{l}\text { Problemas } \\
\text { con las } \\
\text { actividades } \\
\text { cotidianas, } \\
\text { (Si) }\end{array}$ & $-11,8$ & 8,1 & $\begin{array}{c}-27,8 \\
4,1\end{array}$ & $-12,9$ & 8,3 & $\begin{array}{l}- \\
29,3 \\
3,4\end{array}$ & $\overline{18,3}$ & 5,6 & $\begin{array}{l}-35,3 ; \\
-1,4\end{array}$ \\
\hline \multicolumn{10}{|c|}{ Referencia: Sin problemas con dolor/malestar } \\
\hline $\begin{array}{l}\text { Problemas } \\
\text { con } \\
\text { dolor/male } \\
\text { star,(Si) }\end{array}$ & $-6,9$ & 3,6 & $\begin{array}{c}-14,0 \\
0,1\end{array}$ & $-7,5$ & 3,6 & $\begin{array}{l}- \\
14,7 \\
-0,2\end{array}$ & $-6,1$ & 3,7 & $\begin{array}{c}-13,4 \\
1,3\end{array}$ \\
\hline \multicolumn{10}{|c|}{ Referencia: Sin problemas con ansiedad/depresión } \\
\hline $\begin{array}{l}\text { Problemas } \\
\text { con } \\
\text { ansiedad/d } \\
\text { epresión, } \\
\text { (Si) }\end{array}$ & $-3,6$ & 4,3 & $\begin{array}{c}-12,1 \\
4,8\end{array}$ & $-4,0$ & 4,4 & $\begin{array}{l}- \\
12,7 \\
4,6\end{array}$ & $-5,9$ & 4,4 & $\begin{array}{l}-14,6 \\
2,7\end{array}$ \\
\hline \multicolumn{10}{|c|}{ Referencia: Edad $<60$ años } \\
\hline $\begin{array}{l}\text { Edad, ( } \geq 60 \\
\text { Años) }\end{array}$ & & & & 1,5 & 3,1 & $\begin{array}{c}-4,7 \\
7,8\end{array}$ & 2,4 & 3,3 & $\begin{array}{c}- \\
4,2 ; 9 \\
0\end{array}$ \\
\hline \multicolumn{10}{|c|}{ Referencia: Mujeres } \\
\hline $\begin{array}{l}\text { Sexo, } \\
\text { (Hombre) }\end{array}$ & & & & $-6,0$ & 3,3 & $\begin{array}{l}-12,5 \\
0,6\end{array}$ & $-4,5$ & 3,4 & $\begin{array}{c}-11,3 \\
2,3\end{array}$ \\
\hline \multicolumn{10}{|c|}{ Referencia: Nivel socioeconómico bajo } \\
\hline $\begin{array}{l}\text { Nivel } \\
\text { socioeconó } \\
\text { micos, } \\
\text { (Medio) }\end{array}$ & & & & 2,1 & 4,2 & $\begin{array}{l}-6,1 ; \\
10,5\end{array}$ & 4,2 & 4,3 & $\begin{array}{l}-4,3 ; \\
12,6\end{array}$ \\
\hline \multicolumn{10}{|c|}{ Referencia: Grupo atención habitual } \\
\hline $\begin{array}{l}\text { Grupo, } \\
\text { (Interveni } \\
\text { do) }\end{array}$ & & & & 1,8 & 3,4 & $\begin{array}{c}-5,0 \\
8,6\end{array}$ & 1,9 & 3,5 & $\begin{array}{c}-5,1 \\
8,8\end{array}$ \\
\hline \multicolumn{10}{|c|}{ Referencia: DM tipo 2} \\
\hline HTA & & & & & & & $-4,1$ & 6,7 & $\begin{array}{l}-17,7 \\
4,7\end{array}$ \\
\hline HTA/DM2 & & & & & & & 0,3 & 5,4 & $\begin{array}{l}-10,4 \\
10,9\end{array}$ \\
\hline \multicolumn{10}{|c|}{ Referencia: HTA controlada* } \\
\hline $\begin{array}{lr}\text { HTA no } \\
\text { controlada, }\end{array}$ & & & & & & & $-7,1$ & 3,4 & $\begin{array}{l}-13,9 ;- \\
0,3\end{array}$ \\
\hline
\end{tabular}




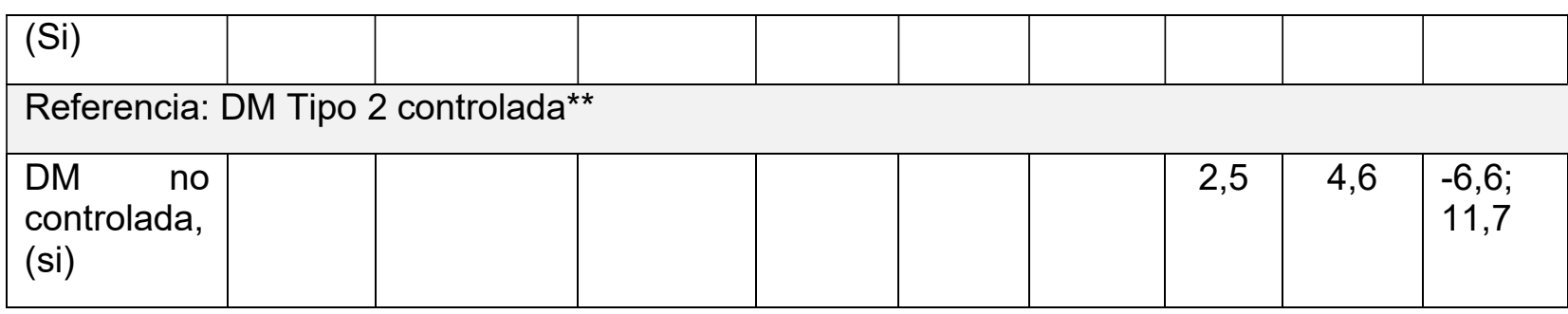

¥Modelo 1: $\boldsymbol{\beta}$ Crudos; ¥Modelo 2: $\beta$ ajustado por edad, sexo, nivel socioeconómico y grupo como variable instrumental; $¥ ¥$ Modelo 3 : $\beta$ ajustado por edad, sexo, nivel socioeconómico, grupo como variable instrumental, enfermedad que padece, control HTA, control DM2. *PAS $<140 \mathrm{mmHg} ;{ }^{* *} \mathrm{HbA} 1_{\mathrm{c}}<7,0 \%$. Fuente: autores.

\section{DISCUSIÓN}

\section{Principales hallazgos}

Las personas con patologías crónicas como la HTA y DM2 enfrentan largos procesos de tratamiento y complicaciones en el transcurso de la enfermedad, por lo cual su CVRS se puede ver afectada. En este sentido, es importante, medir la percepción de las personas acerca de su CVRS, dado que esto nos permite no solo conocer las preferencias ${ }^{(2,3)}$ de los individuos por una serie de estados de salud sino también tomar decisiones clínicas a partir de la generación de indicadores (27). Así, es importante contar con este tipo de información para diseñar e implementar acciones efectivas que mejoren la CVRS de esta población.

En este estudio se encontró que en general los participantes tienen una alta percepción sobre su estado salud, lo que fue evidenciado no solo por los puntajes obtenidos en cada uno de los indicadores del EQ-5D sino también en una mayor frecuencia del estado de salud plena. Así mismo, se encontraron diferencias estadísticamente significativas para la dimensión de actividades cotidianas entre los participantes según la enfermedad que padecen. Finalmente, en el análisis multivariado se hallaron tres factores que disminuyen los puntajes de percepción de la CVRS medida a través de VAS: problemas de dolor/malestar, problemas con actividades cotidianas, y el no control de la HTA.

\section{Comparación con otros estudios}

Con respecto a los estados o perfiles de salud, un estudio (28) halló 169 estados entre una población de individuos con HTA por medio de una encuesta nacional en China, de los cuales el más frecuente fue "salud completa" (11111), con un valor de 68,06\%, y dolor/malestar, hallazgos similares a los de nuestro estudio ${ }^{(28)}$.

En cuanto a las dimensiones del estado de salud, Viegas et al (27) hallaron que la mayoría de los participantes en su estudio no reportaron problemas con el autocuidado (97\%), similar a nuestro hallazgo. En relación con la presencia de problemas en estas dimensiones, dolor/malestar fue la más frecuente, con una prevalencia que oscila entre el $61 \%$ y $38 \%$, especialmente en individuos con DM2 e HTA $^{(14,27)}$. Adicionalmente, se han reportado prevalencias del $30 \%$ para problemas de depresión/ansiedad, similar a las cifras que hallamos ${ }^{(27) .}$ 
Contrario a los hallazgos de nuestro estudio, se han reportado valores más bajos para problemas en movilidad (15,07\%), dolor/malestar (25,96\%), y ansiedad $(10,28 \%)$ en población hipertensa, comparado con población sin diagnóstico para esta patología, diferencias que fueron estadísticamente significativas ${ }^{(28)}$.

En relación a nuestros hallazgos acerca del VAS, autores como Lu et al (13) y Bao et al, ${ }^{(29)}$, encontraron valores que oscilaban entre 80,06 y $76,00 \quad( \pm 13,66)$, respectivamente, en personas con DM2 y HTA, siendo estos valores en el primer caso igual a nuestro estudio, pero menor en el segundo.

En cuanto al índice EQ-5D, cifras entre $0,77(0,75-0,79)$ y $0,95( \pm 0,14)$ en poblaciones con DM2, hipertensión, o ambas patologías, han sido reportadas ${ }^{(14,27)}$, resultados en la mayoría de casos más altos que los hallados en nuestro estudio.

En términos de los factores relacionados que disminuyen la percepción de la CVRS de un individuo, algunos estudios han hallado aspectos que concuerdan con nuestros hallazgos, tales como la presencia de dolor/malestar ${ }^{(14,30)}$, mientras que otros son lo opuesto, como la asociación entre la percepción de CVRS versus multimorbilidad y las afecciones crónicas, donde hay una fuerte correlación entre hipertensión y DM2 $\left(\mathrm{OR}\right.$ ajustado=3,82) e HTA ${ }^{(29)}$. Adicionalmente, otros aspectos han sido asociados con la CVRS de las personas, especialmente en HTA, como la edad, sexo, ${ }^{(28,31,32)}$ e intervenciones educativas ${ }^{\left({ }^{33}\right)}$, los cuales son hallazgos no evidenciados en nuestro estudio.

Aunque la VAS en una herramienta de evaluación ampliamente utilizada, es importante tener en cuenta que es una medida subjetiva de calidad de vida y de muchos otros aspectos.

\section{Fortalezas y limitaciones del estudio}

La principal fortaleza de este estudio fue proporcionar conocimiento acerca de la CVRS en personas con DM2 e HTA en nuestra región, mediante la aplicación de un instrumento genérico usado mundialmente, lo cual nos da una aproximación a la realidad vivida por los pacientes en nuestro contexto de atención primaria. Otra fortaleza es que la muestra de este estudio proviene de un ECA donde se empleó un muestreo probabilístico para la selección de los participantes, lo cual garantiza la representatividad de la población y hace poco probable la existencia de sesgo de selección.

La principal limitación del estudio fue el tamaño de la muestra, que pudo haber conllevado a falta de poder para hallar asociación entre algunas características de los pacientes y su CVRS. Otro aspecto fue la ausencia de medición de otras comorbilidades que podrían haber influido en la percepción de la CVRS de los participantes.

\section{CONCLUSIONES}

La CVRS de personas con DM2 e HTA fue alta, de acuerdo a la evaluación objetiva y subjetiva. No hay evidencia de la existencia de diferencias entre las dimensiones de la CVRS por patología base, con excepción de la dimensión de actividades cotidianas. 
Por otro lado, existen factores que pueden disminuir la percepción de la CVRS del paciente, tales como tener problemas relacionados con las actividades cotidianas, problemas de dolor/malestar, y el no control de la HTA.

\section{Financiación}

Los datos utilizados en este estudio fueron tomados del ensayo clínico aleatorizado "Teaching: Individual to Increase Adherence to Therapeutic Regimen in Hypertension Arterial and / or Diabetes Type 2 (ENURSIN)", el cual fue financiado por COLCIENCIAS CT: 696-2014, código: 617399847755 y por la Universidad Industrial de Santander, Colombia código 617399847755.

\section{Agradecimiento}

A la Gobernación de Santander, Colombia, por la beca académica otorgada a la autora principal de este estudio a través de la convocatoria de Colciencias 771 de 2016.

\section{REFERENCIAS}

1. Miniszewska J, Chodkiewicz J, Zalewska-Janowska A. [Quality of life in health and disease--what is it, how and why evaluate it]. Przegl Lek. 2012;69(6):253-9.

2. Palomino Moral PÁ, Frías Osuna A, del Pino Casado R, Moreno Cámara S. Capítulo 12 - Bienestar: la medición de la calidad de vida relacionada con la salud. En: Martínez Riera JR, del Pino Casado R, editores. Manual Práctico de Enfermería Comunitaria [Internet]. Madrid: Elsevier España; 2014 [citado 28 de octubre de 2020]. p. 59-66.

Disponible

en:

http://www.sciencedirect.com/science/article/pii/B9788490224335000121

3. Herdman M, Badia X, Berra S. El EuroQol-5D: una alternativa sencilla para la medición de la calidad de vida relacionada con la salud en atención primaria. Aten Primaria. 15 de octubre de 2001;28(6):425-30.

4. Haraldstad K, Wahl A, Andenæs R, Andersen JR, Andersen MH, Beisland E, et al. A systematic review of quality of life research in medicine and health sciences. Qual Life Res Int J Qual Life Asp Treat Care Rehabil. octubre de 2019;28(10):2641-50.

5. Salter KL, Moses MB, Foley NC, Teasell RW. Health-related quality of life after stroke: what are we measuring? Int J Rehabil Res Int Z Rehabil Rev Int Rech Readaptation. junio de 2008;31(2):111-7.

6. Khan N, Rahman M, Mitra D, Afsana K. Prevalence of multimorbidity among Bangladeshi adult population: a nationwide cross-sectional study. BMJ Open. 28 de 2019;9(11):e030886.

7. Majumdar UB, Hunt C, Doupe P, Baum AJ, Heller DJ, Levine EL, et al. Multiple chronic conditions at a major urban health system: a retrospective cross-sectional analysis of frequencies, costs and comorbidity patterns. BMJ Open. 15 de 2019;9(10):e029340.

8. Campos-Nonato I, Hernández-Barrera L, Pedroza-Tobías A, Medina C, Barquera S, Campos-Nonato I, et al. Hipertensión arterial en adultos mexicanos: prevalencia, diagnóstico y tipo de tratamiento. Ensanut MC 2016. Salud Pública México. junio de 2018;60(3):233-43.

9. Guarín-Loaiza GM, Pinilla-Roa AE. Adherencia al tratamiento antihipertensivo y su relación con la calidad de vida en pacientes de dos hospitales de Bogotá, D.C. 20132014. Rev Fac Med. 1 de octubre de 2016;64(4):651-7. 
10.Bommer C, Sagalova V, Heesemann E, Manne-Goehler J, Atun R, Bärnighausen T, et al. Global Economic Burden of Diabetes in Adults: Projections From 2015 to 2030. Diabetes Care. 2018;41(5):963-70.

11.Bommer C, Heesemann E, Sagalova V, Manne-Goehler J, Atun R, Bärnighausen $\mathrm{T}$, et al. The global economic burden of diabetes in adults aged 20-79 years: a cost-ofillness study. Lancet Diabetes Endocrinol. 2017;5(6):423-30.

12. Fonseca S, Salvador R, Méndez C, Rafael P, Rodríguez V, Pérez $P$, et al. Calidad de vida en pacientes adultos mayores con Diabetes Mellitus tipo II. 2018;7.

13. Lu Y, Wang N, Chen Y, Nie X, Li Q, Han B, et al. Health-related quality of life in type-2 diabetes patients: a cross-sectional study in East China. BMC Endocr Disord [Internet]. 6 de julio de 2017 [citado 3 de noviembre de 2020];17. Disponible en: https://www.ncbi.nlm.nih.gov/pmc/articles/PMC5501343/

14. Arifin B, Idrus LR, van Asselt ADI, Purba FD, Perwitasari DA, Thobari JA, et al. Health-related quality of life in Indonesian type 2 diabetes mellitus outpatients measured with the Bahasa version of EQ-5D. Qual Life Res Int J Qual Life Asp Treat Care Rehabil. mayo de 2019;28(5):1179-90.

15. Asakura R, Miyatake N, Mochimasu KD, Kurato R, Kuwana S. Comparison of health-related quality of life between type 2 diabetic patients with and without locomotive syndrome. Environ Health Prev Med. septiembre de 2016;21(5):356-60.

16. Reyes MXR, Gomez-Restrepo C, Rodríguez VA, Dennis-Verano R, Kind P. Calidad de vida relacionada con salud en la población Colombiana: ¿cómo valoran los colombianos su estado de salud? Rev Salud Pública. 1 de mayo de 2017;19(3):340-6. 17. Guías de usuario de EQ-5D - EQ-5D [Internet]. [citado 3 de noviembre de 2020]. Disponible en: https://euroqol.org/publications/user-guides/

18. Romero Guevara SL, Parra DI, Rojas LZ. «Teaching: Individual» to increase adherence to therapeutic regimen in people with hypertension and type-2 diabetes: protocol of the controlled clinical trial ENURSIN. BMC Nurs. 4 de junio de 2019;18(1):22.

19. Valuation of EQ-5D - EQ-5D [Internet]. [citado 28 de octubre de 2020]. Disponible en: https://euroqol.org/eq-5d-instruments/valuation-of-eq-5d/

20. Alfonso-Rosa RM, Pozo-Cruz JT del, Daza MC, Pozo-Cruz J del, Pozo-Cruz B del. Asociación entre la condición física relacionada con la salud y la calidad de vida en pacientes diabéticos tipo 2 tratados en atención primaria: un estudio exploratorio en la provincia de Sevilla. Rev Andal Med Deporte. 2012;5(3):91-8.

21. Golicki D, Dudzińska M, Zwolak A, Tarach JS. Quality of life in patients with type 2 diabetes in Poland - comparison with the general population using the EQ-5D questionnaire. Adv Clin Exp Med Off Organ Wroclaw Med Univ. febrero de 2015;24(1):139-46.

22. Grandy S, Fox KM, SHIELD Study Group. Change in health status (EQ-5D) over 5 years among individuals with and without type 2 diabetes mellitus in the SHIELD longitudinal study. Health Qual Life Outcomes. 21 de agosto de 2012;10:99.

23. European Society of Cardiology. Guía ESC/ESH 2018 sobre el diagnóstico y tratamiento de la hipertensión arterial. Rev Esp Cardiol. 1 de febrero de 2019;72(2):160.e1-160.e78.

24. European Heart Journal. 2019 ESC Guidelines on diabetes, pre-diabetes, and cardiovascular diseases developed in collaboration with the EASD | European Heart Journal | Oxford Academic [Internet]. [citado 28 de octubre de 2020]. Disponible en: https://academic.oup.com/eurheartj/article/41/2/255/5556890

25. Ministerio de Salud y Protección Social. Guía de práctica clínica para el diagnóstico, tratamiento y seguimiento de la diabetes mellitus tipo 2 en la población mayor de 18 años. [Internet]. Ministerio de Salud y Protección Social; 2015. Disponible 
en:

http://gpc.minsalud.gov.co/gpc_sites/Repositorio/Conv_637/GPC_diabetes/Guia_Diab etes_Profesionales_Tipo_2.pdf

26. Ministerio de Salud, Colombia. Resolución 008430 , por la cual se establecen las normas científicas, técnicas y administrativas para la investigación en salud [Internet]. Disponible en: https://www.unisabana.edu.co/fileadmin/Documentos/Investigacion/comite_de_etica/ Res_8430_1993_-_Salud.pdf Consultado: enero 04, 2018.

27. Andrade MV, Noronha KVM de S, Maia AC, Kind P. What matters most?: evidence-based findings of health dimensions affecting the societal preferences for EQ-5D health states. Cad Saúde Pública [Internet]. 2013 [citado 30 de enero de 2020];29:s59-72. Disponible en: http://www.scielo.br/scielo.php?script=sci abstract\&pid=S0102311X2013001300006\&lng=en\&nrm=iso\&tIng=en

28. Yao Q, Liu C, Zhang Y, Xu L. Health-Related Quality of Life of People with SelfReported Hypertension: A National Cross-Sectional Survey in China. Int J Environ Res Public Health [Internet]. mayo de 2019 [citado 16 de marzo de 2020];16(10). Disponible en: https://www.ncbi.nlm.nih.gov/pmc/articles/PMC6572246/

29. Bao X-Y, Xie Y-X, Zhang X-X, Peng X, Huang J-X, Du Q-F, et al. The association between multimorbidity and health-related quality of life: a cross-sectional survey among community middle-aged and elderly residents in southern China. Health Qual Life Outcomes. 24 de junio de 2019;17(1):107.

30. Liang Z, Zhang T, Lin T, Liu L, Wang B, Fu AZ, et al. Health-related quality of life among rural men and women with hypertension: assessment by the EQ-5D-5L in Jiangsu, China. Qual Life Res. 1 de agosto de 2019;28(8):2069-80.

31. Kamradt M, Krisam J, Kiel M, Qreini M, Besier W, Szecsenyi J, et al. HealthRelated Quality of Life in Primary Care: Which Aspects Matter in Multimorbid Patients with Type 2 Diabetes Mellitus in a Community Setting? PloS One. 2017;12(1):e0170883.

32. Amer M, Ur-Rahman N, Nazir S-U-R, Jabeen M, Ehsan-Ul-Haq M. sessment Of Blood Pressure and Health-Related Quality of Life Among Hypertensive Patients: An Observational Study. Altern Ther Health Med. mayo de 2019;25(3):26-31.

33. Amer M, Rahman N, Nazir SR, Raza A, Riaz H, Sultana M, et al. Impact of pharmacist's intervention on disease related knowledge, medication adherence, HRQoL and control of blood pressure among hypertensive patients. Pak J Pharm Sci. noviembre de 2018;31(6 (Supplementary):2607-16.

ISSN 1695-6141

(C) COPYRIGHT Servicio de Publicaciones - Universidad de Murcia 